\title{
Subchondral bone influences chondrogenic differentiation and collagen production of human bone marrow-derived mesenchymal stem cells and articular chondrocytes
}

\author{
Michaela Leyh ${ }^{1,2}$, Andreas Seitz ${ }^{3}$, Lutz Dürselen ${ }^{3}$, Jens Schaumburger ${ }^{1}$, Anita Ignatius ${ }^{3}$, Joachim Grifka ${ }^{1}$
} and Susanne Grässel ${ }^{1,2^{*}}$

\begin{abstract}
Introduction: Osteoarthritis $(\mathrm{OA})$ is characterized by an imbalance in cartilage and underlying subchondral bone homeostasis. We hypothesized that signals from the subchondral bone may modulate production of matrix components, alter chondrogenic differentiation potential of cocultured bone marrow-derived mesenchymal stem cells (BMSC) and induce a phenotypic shift in differentiated OA chondrocytes.

Methods: We established a novel coculture model between BMSC, mixed cultures (BMSC and chondrocytes) and chondrocytes embedded in fibrin gel with $O A$ and normal subchondral bone explants ( $O A B$ and NB). Tissues and cells were either derived from OA or trauma patients. In addition, we used adipose-derived stem cells (ASC) from liposuction. With gene expression analysis, biochemical assays, immunofluorescence and biomechanical tests we characterized the properties of newly generated extracellular matrix (ECM) from chondrocytes and chondrogenically differentiating BMSC cocultured with OAB or NB in comparison with monocultures (cultures without bone explants).

Results: Overall, gene expression of collagens of $O A B$ and NB cocultured cells was reduced compared to monocultures. Concomitantly, we observed significantly lower collagen I, II and III and glycosaminoglycan (GAG) production in $O A B$ cocultured cell lysates. In parallel, we detected increased concentrations of soluble GAGs and basic fibroblast growth factor (bFGF), interleukin (IL)-6 and IL-8 in supernatants of OAB and NB cocultures mainly at early time points. IL-1ß concentration was increased in supernatants of OAB cocultures, but not in NB cocultures. Cell-free NB or $\mathrm{OAB}$ explants released different amounts of IL-1B, bFGF and soluble GAG into cell culture supernatants. In comparison to cocultures, monocultures exhibited higher Young's modulus and equilibrium modulus. Stimulation of monocultures with IL-1ß led to a downregulation of aggrecan (ACAN) gene expression and in general to induced matrix metalloprotease (MMP)2, MMP3 and MMP-13 gene expression while IL-6 and IL-8 stimulation partly reduced ACAN, MMP3 and MMP-13 gene expression.
\end{abstract}

Conclusions: Our results suggest an alteration of molecular composition and mechanical properties of the newly formed ECM in subchondral bone cocultures. We suggest that soluble factors, that is interleukins and bFGF, released in cocultures exert inhibitory effects on collagen and temporary effects on proteoglycan production, which finally results in a reduction of mechanical strength of newly formed fibrillar networks.

\footnotetext{
* Correspondence: susanne.graessel@klinik.uni-regensburg.de

'Department of Orthopedic Surgery, University of Regensburg, ZMB/BioPark

1, Josef-Engert-Str. 9, Regensburg 93053, Germany

${ }^{2}$ Centre for Medical Biotechnology, BioPark 1, Josef-Engert-Str. 9, Regensburg

93053, Germany

Full list of author information is available at the end of the article
} 


\section{Introduction}

For long-term repair and regeneration of focal cartilage defects, chondrocytes are implanted at the site of injury, however, not much attention has been paid to the microenvironmental effects of neighboring cartilage/subchondral bone. This is specifically evident in diseases affecting diarthrodial joints such as osteoarthritis (OA), which is an age-related and/or trauma-induced multifactorial, slowly progressing and primarily noninflammatory degenerative disorder of the synovial joints culminating in the irreversible destruction of the articular cartilage [1,2].

Research has focused on chondrocytes and cartilage as mediators of OA but also other cells and tissues of the joint-like synovium or subchondral bone are known to be involved in OA-pathogenesis. There is strong evidence for bone changes during OA progression: increased turnover of subchondral bone, thinning trabecular structures, sclerosis of the subchondral plate, bone marrow lesions and subchondral bone cysts $[3,4]$. Other studies showed alterations in the collagen turnover and cytokine release of osteoarthritic subchondral bone matrix $[5,6]$.

Therapies using adult bone marrow-derived mesenchymal stem cells (BMSC) have a promising future to facilitate regenerative musculoskeletal tissue repair. Especially, BMSC are identified as a relevant cell source for regeneration of focal cartilage and bone lesions, because they can be readily expanded in vitro - whereas differentiated cells, that is chondrocytes dedifferentiate upon expansion [7]. BMSC are pluripotent cells that inherit the capacity to differentiate into cartilage, bone, fat, and other tissue types after appropriate in vitro induction [8].

So far, OA-related cartilage lesions and fissures have not been a widely clinically approved target for BMSCbased therapies as this would imply to implant cells into the neighborhood of diseased tissue where they are confronted with an altered microenvironment of the neighboring pathological cartilage and subchondral bone tissue. It has been demonstrated that BMSC are able to differentiate into a specific cell phenotype depending on the environment they are actually residing in. Crosstalk between BMSC and extracellular matrix (ECM) components could be a crucial determining factor for the differentiation of BMSC into chondrocytes [9]. Indeed, the microenvironment of OA subchondral bone (OAB) is likely to have an influence on the ability of BMSC to regenerate articular cartilage or subchondral bone matrix as implanted stem cells may respond in a different way to differentiation stimuli due to signaling factors secreted from neighboring OA chondrocytes or osteoblasts [10].

One way to direct and redirect the differentiation of BMSC are coculture systems that promote diffusion of secreted paracrine factors and cell-cell interactions $[11,12]$. Westacott et al. demonstrated that subchondral osteoblasts are able to modulate the metabolism of chondrocytes and change their phenotype [13]. Of note, the ratio of cocultured BMSC and articular chondrocytes regulate whether differentiation proceeds toward a cartilaginous or osseous phenotype. Culturing articular chondrocytes with BMSC in a 2:1 ratio induces both phenotypes simultaneous in a three-dimensional alginate hydrogel construct indicating that chondrocytes provide the necessary factor(s) in this process [14]. These osteoinductive properties of articular chondrocytes were also reported for a culture model where chondrocytes in alginate hydrogels were cocultured above a monolayer of BMSC preventing direct cell-to-cell contact. Notably, the effect was time-dependent, the longer the coculture period the more prolonged and stable was the osteogenic phenotype of BMSC [15].

However, the underlying mechanisms of cell-cell interactions in OA joint tissues that influence chondro-osteogenic differentiation of BMSC have not been elucidated nor fully understood. Also, the identity of factors from OAB bone tissue and cells that might modulate the chondrogenic phenotype of BMSC is not comprehensively delineated. We address this subject in the present study where we have established a novel in vitro coculture model to evaluate the influence of subchondral bone from OA-affected joints on chondrogenic differentiation of human BMSC derived from OA patients, the phenotype of differentiated OA chondrocytes and the properties of newly synthesized ECM. In addition, we have included a 'triculture' model consisting of a mixture of chondrocytes and BMSC cultured on subchondral bone explants. In order to attribute effects to disease status and cell source, we reproduced key experiments using a coculture regimen with adiposederived stem cells and 'normal' subchondral bone explants from trauma patients. Here, we aim to test if cell-to-cell contact between differentiated chondrocytes and undifferentiated BMSC compensate effects of factors from subchondral bone on chondrogenic differentiation of BMSC. To provide a chondrogenic favorable environment, cells were embedded in fibrin gel [16], seeded onto the surface of subchondral bone explants and kept in chondrogenic medium for up to 28 days.

\section{Material and methods}

\section{Culture and isolation of human subchondral bone explants, BMSC and chondrocytes}

Human articular cartilage was collected from surgically removed joints of patients undergoing total knee replacements (TEP) due to OA. This had been approved by the local ethics committee (Az: 08/065; Ethikkommission an der Universität Regensburg, email: ethikkommission@klinik.ukr.de) and specimens were taken with patients' written consent. For this study, knee joints were obtained from 32 different donors (13 male and 19 female, mean age $67 \pm 9$ ). Prior to culture, the cartilage 
surface of surgically removed tissue was classified macroscopically as either damaged or intact according to a predefined procedure comprising color, surface integrity and tactile impression tested with a standard scalpel [17]. We used only healthy-appearing pieces for isolation of chondrocytes and generation of subchondral bone chips. $\mathrm{OAB}$ chips were produced as follows: surgically removed tissue was thoroughly washed with phosphate-buffered saline (PBS). Then the cartilage surface was scored as described above and only justly evaluated pieces were accepted for usage. Cartilage was cut off for isolation of chondrocytes and the completely denuded bone was used for generation of explants of $8 \mathrm{~mm}$ in diameter and $4 \mathrm{~mm}$ height. Chondrocytes were isolated from cartilage slices upon overnight digestion with collagenase II (PAA, Piscataway, NJ, USA) at $37^{\circ} \mathrm{C}$. Isolated cells were resuspended in Dulbecco's modified Eagle's medium (DMEM; Gibco Invitrogen, Paisley, UK) containing $10 \%$ fetal calf serum (FCS; SigmaAldrich, St. Louis, MO, USA) and $1 \%$ penicillin/streptomycin (PAA, Piscataway, NJ, USA). Chondrocytes were kept in monolayer in an initial density of 17,000 cells per $\mathrm{cm}^{2}$ cultured in a humidified $37^{\circ} \mathrm{C} / 5 \% \mathrm{CO}_{2}$ incubator for 7 to 14 days and were used when confluent (passage 1). Normal subchondral bone tissue (NB) was received from knee joints of rare trauma-affected patients (treated for sport accidents, two male and two female, mean age $35 \pm 11$ ) and were prepared as described above. The bone tissue used in this study was not injured directly but was collected during the surgical treatment of the patient from another nonaffected region of the same joint. Preparation of bone explants for culture was similar for OA- and trauma-derived bone tissue.

All human BMSC used for this study have been isolated from bone marrow aspirates obtained from a total number of 30 different patients (14 male and 16 female, mean age $61 \pm 8$ ) undergoing a hip replacement surgery due to OA. This had been approved by the local ethics committee (Az: 08/065; Ethikkommission an der Universität Regensburg, email: ethikkommission@klinik.ukr.de) and specimens were taken with patients' written consent. The bone marrow was centrifuged and cells were fractionated on a density gradient (Biocoll separating solution; Biochrom, Berlin, Germany). The low-density cell fraction concentrated in the interphase ('buffy coat') was washed, seeded in cell culture flasks supplied with MesenchymStem Medium (PAA, Piscataway, NJ, USA) and nonadherent cells were removed after 5 to 7 days. Adherent cells were cultured until they reached approximately $80 \%$ confluence. After splitting, BMSC were seeded at a density of $4 \times 10^{4}$ cells $/ \mathrm{cm}^{2}$ and kept in culture for up to three passages before they were used for experiments. At this passage, BMSC were positive for CD44 and CD105 and negative for CD19 and CD34. Additionally, we analyzed their differentiation capacity into osteogenic and adipogenic lineages.
A representative specimen is shown in Figure S2 in Additional file 1.

Adipose-derived stem cells (ASC) (isolated as described previously by [18]) were isolated from subcutaneous fat tissue that was obtained from patients undergoing elective body-contouring procedures. Five different patients were used for our coculture setup (female, age <50). Written consent to harvest ASC from patients was obtained from the local ethics committee (Az: 08/117; Ethikkommission an der Universität Regensburg, email: ethikkommission@ klinik.ukr.de).

Briefly, fat tissue was washed, minced and digested in serum-free MEM ( $1 \mathrm{ml} / 1 \mathrm{~g}$ tissue) with Liberase Blendzyme 3 (2U/1 g tissue; Roche Diagnostics, Basel, Switzerland) at $37^{\circ} \mathrm{C}$ for $45 \mathrm{~min}$. The lysate was filtered (100- and 40- $\mu \mathrm{m}$ filters; Fisher Scientific, Schwerte, Germany) and centrifuged at $450 \mathrm{x}$ g for $10 \mathrm{~min}$. The cell pellet was washed twice with Hanks' balanced salt solution (Cellgro, Manassas, VA, USA) and cells were seeded in culture vials (Greiner Bio-one, Frickenhausen, Germany) and daily washed to remove unwanted red blood cells or nonadherent cells. After reaching a confluence of $80 \%$, ASC were seeded at a density of 3,000 cells $/ \mathrm{cm}^{2}$, maintained in ASC medium ( $\alpha$ MEM containing 20\% FCS, $2 \mathrm{mM} \mathrm{L-glutamine}$ and $1 \%$ penicillin/streptomycin, SigmaAldrich, St. Louis, MO, USA) and used for experiments at passages 5 .

ASC were characterized in accordance with guidelines of the Declaration of Helsinki for biomedical research from the Applied Stem Cell Research Center of the University of Regensburg.

A suspension of fibrinogen $(10 \mu \mathrm{l}, 100 \mathrm{mg} / \mathrm{ml}$, SigmaAldrich, St. Louis, MO, USA) and $1 \times 10^{6}$ BMSC, $1 \times$ $10^{6}$ ASC, $2 \times 10^{6}$ chondrocytes or a mixture of $5 \times 10^{5}$ BMSC and $5 \times 10^{5}$ chondrocytes (1:1) was homogenously mixed with thrombin $(18 \mu \mathrm{l}, 5 \mathrm{U} / \mathrm{ml}$; Baxter, Munich, Germany). Because of the smaller size of chondrocytes, their cell number had to be adjusted to obtain a comparable volume of fibrin gel and to avoid effects due to different medium diffusion capacity resulting in an altered maintenance with nutrients. The cell-fibrinogen suspension was applied onto the surface of either $\mathrm{OAB}$ or $\mathrm{NB}$ explants (co- and tricultures) or as a droplet on the bottom of a 24-well plate (monocultures) (Figure 1). Full polymerization was reached after $45 \mathrm{~min}$ at $37^{\circ} \mathrm{C}$ and resulted in a stable and clear hydrogel with a pore size of approximately $50 \mu \mathrm{m}$. Cell-free $\mathrm{OAB}$ and NB explants were included into our setups. Mono-, co- and tricultures as well as cell-free bone explants were kept in chondrogenic medium in the presence of transforming growth factor $ß$ (TGF-ß)-3 (10 ng/ml, R\&D Systems, Minneapolis, MN, USA) and cultured for up to 4 weeks [19]. After 7 and 28 days, a specimen of co- or tricultured fibrin gels was carefully separated from subchondral bone explants 


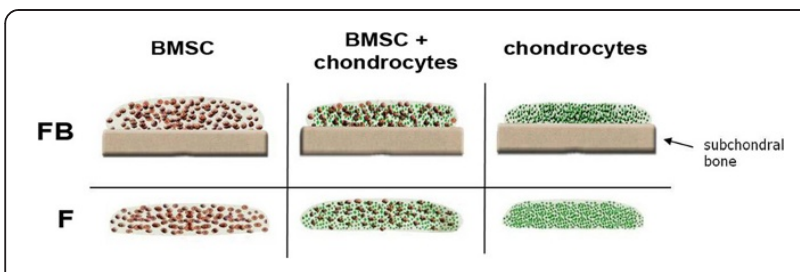

Figure 1 Model of contact coculture between cells and subchondral bone explants. BMSC, mixed cultures (BMSC + chondrocytes 1:1) and chondrocytes were embedded in fibrin gel and these cell-gel constructs were applied onto the surface of subchondral bone explants (co- and tricultures). As controls, cells were embedded in fibrin gels and cultured without subchondral bone (monocultures). All experimental setups were kept for up to 28 days in chondrogenic medium. Samples were harvested at days 7 and 28. BMSC, bone marrow-derived mesenchymal stem cells; F, fibrin gel-embedded monocultures (without subchondral bone explants); FB, fibrin gel-embedded co- and tricultures together with subchondral bone explants.

using a thin spatula. Fibrin gels were processed for histology, immunofluorescence, biochemistry, biomechanical or gene expression analysis as described below. Culture supernatants of day 7 and 28 were collected and frozen at $-80^{\circ} \mathrm{C}$ until usage.

\section{Cell vitality assay in fibrin gel setups}

In order to evaluate vitality of cells in our different culture setups, lactate dehydrogenase (LDH) concentration in supernatants of BMSC, mixed cultures and chondrocytes was determined in monocultures and co- or tricultures with $\mathrm{OAB}$ kept in chondrogenic medium. Content of LDH was analyzed at days 7, 14, 21 and 28 with an LDH-based cytotoxicity detection kit (Roche Diagnostics, Penzberg, Germany) and compared to respective assay controls (high control = all cells in fibrin gels were lysed; low control $=$ spontaneous cell death of an equivalent cell amount in monolayer) according to the manufacturer's instructions. LDH concentration released from dead cells into supernatant was determined on a photometrical basis at absorption of $490 \mathrm{~nm}$ (Tecan GENios with Magellan 6.5; Tecan, Crailsheim, Germany). Due to high interexperimental variability - presumably caused by personal living conditions and physical activity, medical treatment or general health status of tissue donors - we have calculated the raw data as percentage of control per individual experiment. Repetition was in triplicate at least three times with cells and explants from different donors.

\section{Histology and immunofluorescence of fibrin gel- embedded cultures}

Fibrin gels were rinsed with PBS (PAA, Piscataway, NJ, USA), embedded in TissueTec (Sakura Finetek, Alphen aan den Rijn, the Netherlands) and were frozen in liquid nitrogen. For histological evaluation, sections $(10-\mu \mathrm{m}$ thick) were fixed in $4 \%$ paraformaldehyde (PFA: Sigma-Aldrich,
St. Louis, MO, USA) for $10 \mathrm{~min}$ at RT, stained with $1 \%$ Alcian blue 8GX (Sigma-Aldrich, St. Louis, MO, USA) for glycosaminoglycans (GAGs) and counterstained with nuclear fast red aluminium sulfate solution (Roth, Arlesheim, Germany). For immunofluorescence analysis, sections were fixed in 4\% PFA for $10 \mathrm{~min}$ at RT, treated for $30 \mathrm{~min}$ at $37^{\circ} \mathrm{C}$ with pepsin $(3 \mathrm{mg} / \mathrm{ml}$ in $0.01 \mathrm{M} \mathrm{HCl}$, Sigma-Aldrich, St. Louis, MO, USA) and were blocked with $3 \%$ bovine serum albumin (BSA) (Sigma-Aldrich, St. Louis, MO, USA) diluted in PBS for $1 \mathrm{~h}$. Sections were labeled overnight at $4^{\circ} \mathrm{C}$ with an antibody against collagen I (C-2456, Sigma-Aldrich, St. Louis, MO, USA), collagen II (CIIC1; DSHB, Iowa City, IA, USA) or collagen III (MAB 3392; Merck Millipore, Billerica, MA, USA). In case of collagen X staining (2031501005; Quartett, Berlin, Germany) a hyaluronidase (Sigma-Aldrich, St. Louis. MO, USA) pretreatment instead of pepsin was used. For fluorescence detection, all sections were incubated with an Alexa Fluor ${ }^{\text {Tw }}$ 488 secondary antibody (Invitrogen, Paisley, UK) for $1 \mathrm{~h}$ at $37^{\circ} \mathrm{C}$. Finally, samples were mounted in Vectashield mounting medium with DAPI $(1 \mu \mathrm{g} / \mathrm{ml}$, Vector Laboratories, Burlinton, ON, Canada) and were analyzed with an Olympus BX 61 imaging system and cell ${ }^{\mathrm{P}}$ software (Olympus, Hamburg, Germany). Repetition was at least five times with cells and explants from different donors.

\section{Biochemical analysis of fibrin gel cell lysates and supernatants}

Fibrin gels were homogenized and digested with pepsin $(1 \mathrm{mg} / \mathrm{ml}$ in $0.5 \mathrm{M}$ acetic acid containing $0.4 \mathrm{M} \mathrm{NaCl}$, Sigma-Aldrich, St. Louis, MO, USA) for $48 \mathrm{~h}$ at $4^{\circ} \mathrm{C}$ and further digested with elastase $(1 \mathrm{mg} / \mathrm{ml}$ in Tris-buffered saline (TBS) pH 8; Serva Electrophoresis, Heidelberg, Germany) for $24 \mathrm{~h}$ at $4^{\circ} \mathrm{C}$. Samples were stored at $-20^{\circ} \mathrm{C}$ until they were analyzed for GAGs and collagen contents.

GAG concentration was measured spectrophotometrically using $25 \mu \mathrm{l}$ of the digested cell lysates or $25 \mu \mathrm{l}$ undiluted cell supernatant supplemented with dimethylmethylene blue (DMMB; AppliChem, Darmstadt, Germany), which forms a complex with GAGs. Quantification was performed in $\mu \mathrm{g}$ per $1 \times 10^{6}$ cells with a chondroitin sulfate standard at $525 \mathrm{~nm}$ (Tecan GENios with Maggelan 6.5; Tecan, Crailsheim, Germany).

Collagen I and II contents of day 28 cell lysates digested as described above were measured with specific sandwich enzyme-linked immunosorbent assays (ELISAs), which recognize the native conformation of collagen I and II chains (Chondrex, Redmond, WA, USA), according to the manufacturer's instructions.

Collagen III in digested cell lysates was quantified with a dot blot assay using $1 \mu \mathrm{l}$ from $500 \mu \mathrm{l}$ of cell lysate and including a standard curve of recombinant collagen III (Abcam, Cambridge, UK). Quantification was performed densitometrically in $\mu \mathrm{g}$ per $1 \times 10^{6}$ cells using a Chemi- 
Smart 500 (PeqLab, Erlangen, Germany) for fluorescence detection and the CS4 Windows software for calculation of luminescence intensities. Repetition was in triplicate at least five times with cells and explants from different donors.

\section{RNA isolation and real-time PCR amplification}

Cell-fibrin gel suspensions suspended in peqGOLD TriFast (PeqLab, Erlangen, Germany) were minced and RNA was isolated according to a Trizol protocol, followed by column purification with the absolutely RNA Microprep Kit (Agilent Technologies Stratagene, Santa Clara, CA, USA) according to the manufacturer's instructions. cDNA was generated from 500 ng of RNA using AffinityScript QPCR cDNA Synthesis Kit and oligo(dT) primers (Agilent Technologies Stratagene, Santa Clara, CA, USA) according to the manufacturer's instructions. Repetition was in triplicate at least five times for BMSC, mixed and chondrocytes cocultures with bone explants from different OA donors and at least four times for ASC cultures.

Quantitative real-time PCR was performed in triplicate using 30 ng cDNA (RNA equivalent) and qPCR master mix SYBR Green Dye I on MxPro-Mx305P (Agilent Technologies Stratagene, Santa Clara, CA, USA). For quantification, a plasmid standard curve for COL1A1, COL2A1, COL3A1 and COL1OA1 was included on each PCR plate (plasmid copy ranges for COL1A1: $1 \times 10^{6}$ to $1 \times 10^{2}$; COL2A1: $3.4 \times 10^{7}$ to $3.4 \times 10^{3}$; COL $3 A 1: 1 \times 10^{6}$ to $1 \times 10^{2}$ and COL1OA1: $5 \times 10^{4}$ to $\left.5 \times 10^{0}\right)$. Gene expression of matrix metalloproteinase $(M M P) 2, M M P 3$, $M M P 13$ and aggrecan $(A C A N)$ gene was normalized with GAPDH and data evaluation was relative employing the $\triangle \triangle C T$ method using gene expression of unstimulated monocultures as calibrator. Data analysis was carried out with the MxPro QPCR 4.0 software (Agilent Technologies Stratagene, Santa Clara, CA, USA). The following forward and reverse primer pairs were used for gene expression analysis: for COL1A1 5'-AGC TCC TGG TGA AGT TGG TC-3' and $5^{\prime}$-ACC AGG GAA GCC TCT CTC TC-3'; for COL2A1 5' -TGC TGC CCA GAT GGC TGG AAG A-3' and $5^{\prime}$-TGC CTT GAA ATC CTT GAG GCC C-3'; for COL3A1 5'-GTC CAT GGA TGG TGG TTT TC $-3^{\prime}$ and $5^{\prime}$-GTG TGT TTC GTG CAA CCA TC-3'; for COL10A1 5'-CCC TCT TGT TAG TGC CAA CC-3' and $5^{\prime}$-AGA TTC CAG TCC TTG GGT CA-3'; for MMP2 5'-GCC AAT GGA GAC TGT CTC AAG A-3' and 5'-TTC TAA GGC AGC CAG CAG TGA A-3'; for MMP3 5'-AAC CTG TCC CTC CAG AAC CT-3' and 5'-GGAAGAGATGGCCAAAATGA-3'; for MMP13 5'-CAC CGG CAA AAG CCA CTT-3'and 5'-TAGAC TGG TAA TGG CAT CAA GGG A-3'; for ACAN 5'-CTA TAC CCC AGT GGG CAC AT-3' and 5'-GGC ACT TCA GTT GCA GAA GG-3' and for GAPDH 5'-ACC CAG

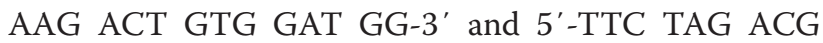
GCA GGT CAG GT-3'.

\section{Analysis of soluble collagens in culture supernatants with hydroxyproline assay}

The amount of total soluble collagen in culture supernatants was determined by the Total Collagen Hydroxyproline Assay (QuickZyme Biosciences, Leiden, Netherlands) according to the manufacturer's protocol. Briefly, after 3 days, $1 \mathrm{ml}$ culture supernatant from monocultures or subchondral bone cocultures was harvested and soluble collagens in the supernatant were hydrolyzed into amino acids $\left(12 \mathrm{M} \mathrm{HCl}\right.$ for $20 \mathrm{~h}$ at $95^{\circ} \mathrm{C}$ ). Hydroxyproline was stained and color formation was quantified at absorbance maximum at $570 \mathrm{~nm}$ (Tecan GENios with Maggelan 6.5; Tecan, Crailsheim, Germany). Repetition was in triplicate at least five times with cells and explants from different donors.

\section{Analysis of culture supernatants for bFGF, IL-1ß, IL-6 and IL-8}

To determine the concentration of specific proteins in the supernatant, human interleukin-1ß (IL-1ß) sandwich ELISA kit (Ray Biotech, Inc., Norcross, GA, USA), interleukin-6 (IL-6) sandwich ELISA kit (R\&D Systems, Minneapolis, MN, USA), interleukin-8 (IL-8) ELISA kit (Gen-Probe, Bedford, MA, USA) and basic fibroblast growth factor (bFGF) sandwich ELISA kit (R\&D Systems, Minneapolis, MN, USA) were used according to the manufacturer's instructions. Repetition was in triplicate at least six times for BMSC, mixed cultures and chondrocytes cocultured with bone explants from different OA donors and at least four times for cell-free OA and normal bone explants as well as for ASC cultures.

\section{Biomechanical testing}

Samples with a standardized outer diameter of $2.6 \mathrm{~mm}$ were punched out of the fibrin gel-cell constructs (biopsy punch, Stiefel, Munich, Germany). The biomechanical tests were carried out in a standard material testing machine (Z010; Zwick, Ulm, Germany) equipped with a $40 \mathrm{~N}$ load cell. The initial sample height $\left(\mathrm{h}_{0}\right)$ was assessed under a preload of $0.1 \mathrm{~N}$ by use of a laser displacement transducer (optoNCDT 2200-20; Micro-Epsilon Messtechnik $\mathrm{GmbH} \&$ Co., Ortenburg, Germany, $0.3 \mu \mathrm{m}$ resolution, $\pm 0.03 \%$ accuracy). Subsequently, the samples were placed in a cell culture dish filled with $0.9 \% \mathrm{NaCl}$ and an unconfined compression test was performed by loading it through a flat-ended punch at a strain rate of $100 \% \mathrm{~h}_{0} / \mathrm{min}$ until $50 \%$ strain was reached. The Young's modulus was then calculated at two typical regions from the stress-strain diagrams (progressive region at 0 to $10 \%$ strain and the linear region at 40 to $50 \%$ strain). The samples were then stored for $24 \mathrm{~h}$ at $4^{\circ} \mathrm{C}$ in physiological saline to let them 
completely recover. After that, an additional stress-relaxation test was performed. The samples were placed in a confining chamber of $2.6 \mathrm{~mm}$ diameter filled with $0.9 \%$ $\mathrm{NaCl}$ and loaded by a porous ceramic cylinder $\left(\mathrm{Al}_{2} \mathrm{O}_{3}\right)$ allowing for fluid exudation. Fifty percent strain was applied at a strain rate of $100 \% \mathrm{~h}_{0} / \mathrm{min}$ and held constant for $10 \mathrm{~min}$ until the equilibrium state was reached. Then the hydraulic permeability $(\mathrm{k})$ was determined using a least squares algorithm derived from a diffusion equation $[20,21]$ (Formula 1 ), where $\Delta \mathrm{l} / \mathrm{h}_{0}$ is the applied strain and $t$ the time. The aggregate modulus $\left(\mathrm{H}_{\mathrm{A}}\right)$ at equilibrium state at $50 \%$ strain was calculated as described in Formula 2 where $\sigma_{\infty}$ was the equilibrium stress at a strain of $50 \%$. Repetition was four times with cells and explants from different donors.

$$
\begin{aligned}
& \text { Formula } 1 \sigma_{\infty}+2 H * \frac{\Delta 1}{h_{0}} * e^{\left(\left(\frac{\pi}{h_{0}}\right)^{2} * H * K * t\right)} \\
& \text { Formula 2 } H_{A}=\frac{\sigma_{\infty}}{\varepsilon_{50 \%}}
\end{aligned}
$$

\section{Stimulation of BMSC, mixed cell populations and chondrocytes embedded in fibrin gel}

Stimulation of monocultures with IL-1ß $(5 \mathrm{ng} / \mathrm{ml}$, Biomol, Hamburg, Germany), IL-6 (5 ng/ml, RayBiotech, Norcross, GA, USA) or IL-8 (10 ng/ml, RayBiotech, Norcross, GA USA) was performed in chondrogenic medium for the first 7 days. Fibrin gels were harvested after 7 or 28 days and processed for gene expression analysis as described above. Repetition was in triplicate at least five times with cells and bone explants from different donors.

\section{Culture setups of ASC with subchondral bone}

ASC were seeded onto the surface of OAB or NB explants as described above. Fibrin gels of days 7 and 28 were processed for qPCR and culture supernatants were collected and frozen at $-80^{\circ} \mathrm{C}$ until usage for analysis of cytokines, bFGF and GAG.

\section{Stimulation of BMSC with conditioned medium}

BMSC monocultures were supplemented either with fresh chondrogenic medium or with chondrogenic medium that was conditioned for three days by $\mathrm{OAB}$ explants. Monocultures and stimulated cultures were kept under these conditions for up to 28 days and medium was changed every 3 days. After 7 and 28 days, fibrin gels were processed for gene expression analysis as described above. Repetition was in triplicate at least four times with cells and explants from different donors.

\section{Statistical analysis}

All results are calculated for an initial cell number of $1 \times 10^{6}$ cells. The mean standard deviation (SD) values were calculated for all variants. The nonparametric Wilcoxon test (for paired analyses) or the Mann-Whitney test (unpaired analyses) was applied to analyze differences between time points and between culture conditions. All experiments were performed in triplicate and repeated at least four times with cells from different donors. $P<0.05$ values were considered to indicate statistically significant differences. Data analysis and graphing was performed with GraphPad for Windows version 5 (GraphPad Software, Inc., La Jolla, CA, USA).

\section{Results}

\section{Viability of cultured cells}

In order to evaluate if the fibrin gel system affects vitality/viability of cells, LDH concentration in culture supernatants was analyzed and compared to respective monolayer controls. Chondrocytes, BMSC and mixed cultures embedded in fibrin gel cocultured or kept as monocultures showed no enhanced LDH release compared to assay controls. We therefore assume that cells are not influenced in their vitality or overall metabolic activity by fibrin gel components or subchondral bone explants during the culture time period (Figure S1A-C in Additional file 2).

\section{Culture models}

We set up different culture conditions to analyze the influence of factors from subchondral bone on multipotent BMSC with respect to ECM formation and chondrogenic differentiation. Therefore, we cocultured BMSC embedded in fibrin gel with subchondral bone explants from OA patients. As a control for chondrogenic properties of BMSC we used cocultures of differentiated articular chondrocytes and mixed cultures (tricultures) of chondroctyes and BMSC (both embedded together in fibrin gels) to include putative cell-to-cell effects of differentiated cells on undifferentiated cells. As a control for cocultures we set up the respective monocultures of cells embedded in fibrin gels and cultured in chondrogenic medium (Figure 1). Additionally, we analyzed cell-free subchondral bone explants from OA and trauma donors with respect to cytokines, soluble (s)GAG and bFGF release and included an ASC coculture set up with $\mathrm{OAB}$ and $\mathrm{NB}$ explants with respect to gene expression and release of cytokines, sGAG and bFGF.

\section{Collagen gene expression of cocultures versus monocultures}

To determine whether factors released from subchondral bone $(\mathrm{OAB}$ or $\mathrm{NB})$ affect matrix production or chondrogenic differentiation of ASC or BMSC and chondrogenic phenotype of chondrocytes, mRNA expression of COL1A1 
(dedifferentiation marker), COL2A1 (marker for chondrogenic differentiation), COL3A1 (mesenchymal marker) and COL10A1 (marker for chondrocyte hypertrophy) was analyzed at days 7 and 28 .

At day 7 , we observed a significant inhibition of COL1A1 gene expression in ASC and BMSC cocultured with $\mathrm{OAB}$ and in mixed tricultures compared to monocultures, whereas ASC cocultured with NB and chondrocyte $\mathrm{OAB}$ cocultures remained unaffected. At day 28 none of the culture conditions that affected COL1A1 gene expression was statistically significant. ASC cocultured with NB had higher mRNA level of COL1A1 compared to mixed tricultures at day 28 while all other $\mathrm{OAB}$ coculture conditions revealed no significant difference to NB cocultures (Figure 2A). Analyses of COL2A1 gene expression in $\mathrm{OAB}$ cocultures revealed a significant upregulation in ASC cocultures and a significant downregulation in BMSC cocultures compared to respective monocultures at day 28. COL2A1 gene expression in all other culture regimen remained unaltered. No significant differences in COL2A1 gene expression were detectable between $\mathrm{NB}$ and $\mathrm{OAB}$ cocultures (Figure 2B).

In all co- and tricultures COL3A1 gene expression was significantly downregulated at day 7 and in mixed tricultures and chondrocyte cocultures also at day 28 compared to monocultures. No significant differences in COL3A1 gene expression were detectable between $\mathrm{NB}$ and $\mathrm{OAB}$ cocultures (Figure 2C).

COL10A1 gene expression was reduced in NB cocultures with ASC at day 28 and in OAB cocultures with ASC and BMSC at day 7 and in BMSC and chondrocyte cocultures at day 28 compared to respective monocultures. In ASC cocultured with NB, COL10A1 gene expression was increased compared to $\mathrm{OAB}$ cocultures

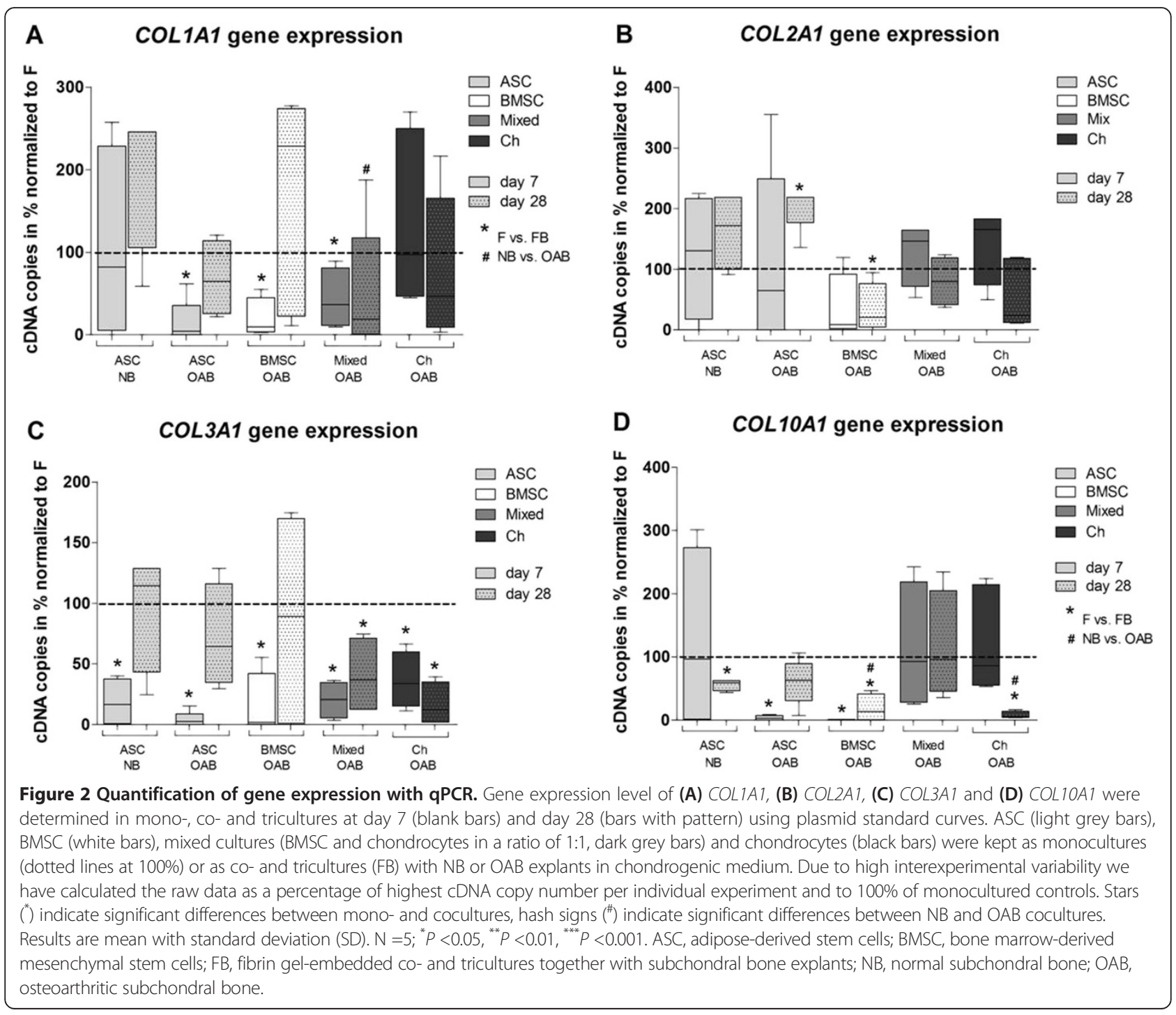


of BMSC and chondrocytes at day 28, while all other $\mathrm{OAB}$ coculture conditions revealed no significant difference to NB cocultures (Figure 2D).

Overall, we observed an inhibitory effect of OAB explants on collagen gene expression in co- and tricultures whereas NB explants affected collagen gene expression less.

\section{Quantification of collagen and proteoglycan synthesis in cell lysates}

In further experiments, we studied the influence of $O A B$ explants on collagen (day 28) and GAG/proteoglycan (days 7 and 28) synthesis.

We detected a significantly reduced collagen I production in BMSC cocultures and mixed tricultures in comparison to monocultures. In chondrocytes, collagen I synthesis was below assay detection limit in both culture conditions (Figure 3A). We observed a significantly reduced collagen II production in BMSC and chondrocyte cocultures compared to monocultures while mixed tricultures remained unaffected (Figure 3B). Collagen III production in all three co- and triculture conditions was significantly reduced compared to monocultures (Figure 3C). A dimethylmethylene blue (DMMB) assay demonstrated that all co- and triculture cell lysates had a significantly decreased GAG/proteoglycan content on day 7 (Figure 3D) while on day 28 no significant differences were detected between mono-, co- and tricultures (data not shown).

Overall, we observed an inhibitory effect of $O A B$ on collagen and proteoglycan synthesis in co- and tricultured cells.

\section{Immunofluorescence staining of collagens and histological staining of GAG}

Collagen deposition into the ECM of mono-, co- and tricultures was demonstrated by immunofluorescence of cryosections at day 28.

BMSC and mixed monocultures deposited a higher amount of collagen I into their ECM than those co- or

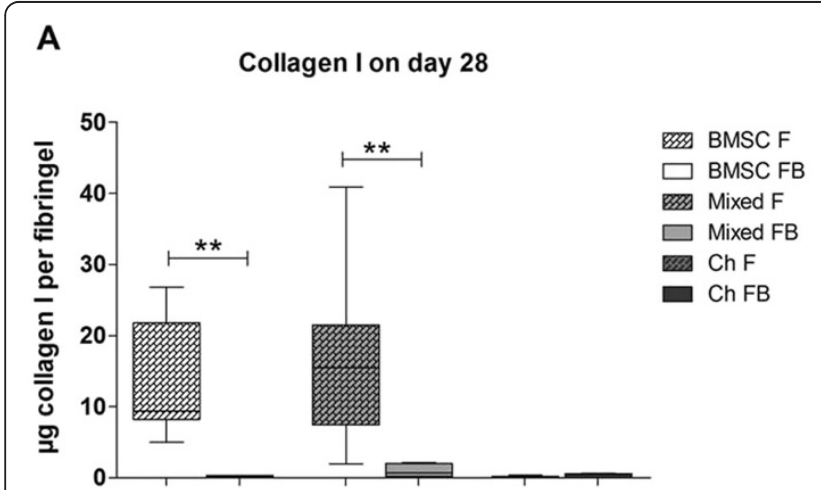

\section{B}

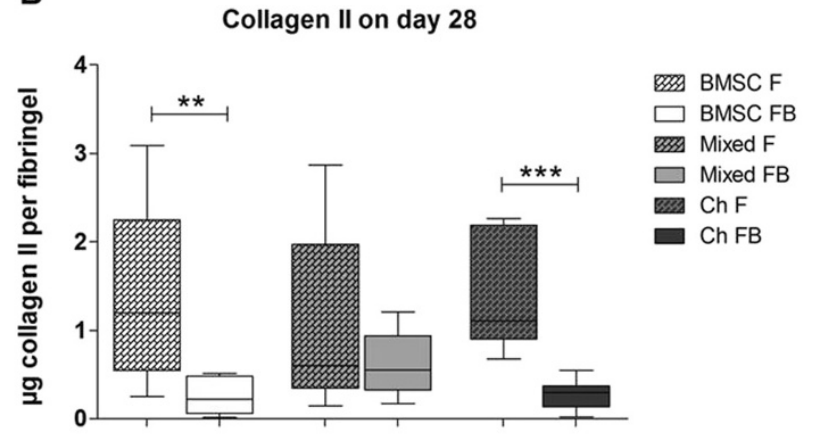

C

Collagen III on day 28

D

GAG on day 7
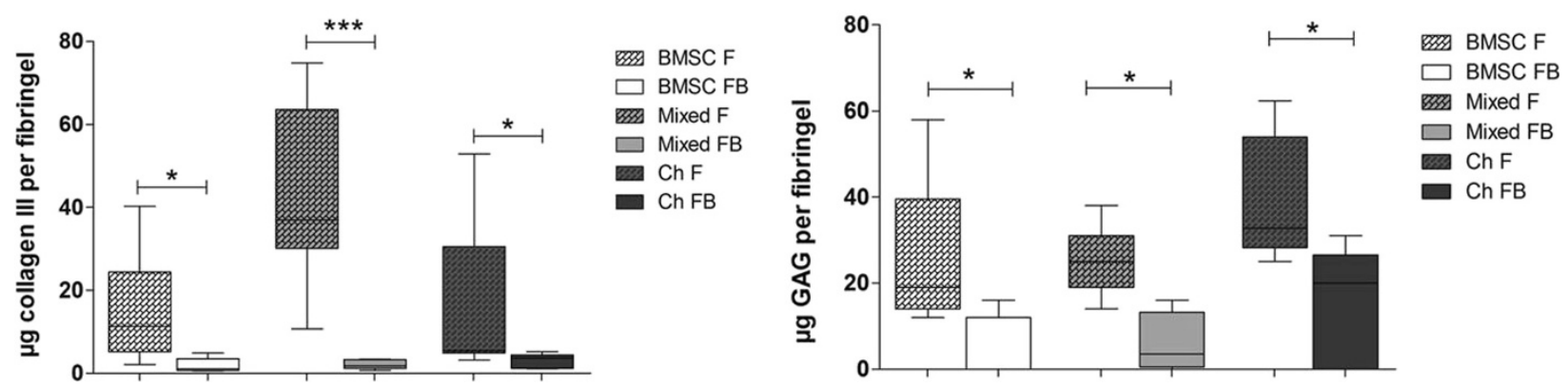

Figure 3 Quantification of collagens I, II, III and proteoglycans in cell lysates. Protein synthesis of collagens I (A), II (B) and III (C) in cell lysates after 28 days of culture in fibrin gels and GAGs (D) in cell lysates on day 7. BMSC (white bars), mixed cultures (BMSC and chondrocytes in a ratio of 1:1, grey bars) or chondrocytes (black bars) were embedded in fibrin gel and kept in monoculture (F, bars with pattern) or in co- and triculture with $\mathrm{OAB}$ explants ( $\mathrm{FB}$, blank bars) in chondrogenic medium. (A, B) Collagens I and II were quantified with ELISA, or (C) by densitometrically evaluated dot-blot analysis containing a recombinant collagen III standard curve. (D) Proteoglycan/GAG concentration in cell lysates was quantified by a DMMB assay including a chondroitin sulfate standard curve. Results are mean with standard deviation (SD). N $=5 ;{ }^{*} P<0.05,{ }^{* * *} P<0.01,{ }^{* * *} P<0.001$. BMSC, bone marrow-derived mesenchymal stem cells; DMMB, dimethylmethylene blue; $F$, fibrin gel-embedded monocultures (without subchondral bone explants); FB, fibrin gel-embedded co- and tricultures together with subchondral bone explants; GAG, glycosaminoglycan; OAB, osteoarthritic subchondral bone. 
tricultured with OAB explants. Staining for collagen I in chondrocytes was poor in both culture conditions (Figure 4A). Staining for collagen II was detected in all monoculture conditions and poor or no staining in co- and tricultures with $\mathrm{OAB}$ explants (Figure 4B). We observed similar collagen III staining in all monoculture conditions but only little or no staining in the co- and triculture setups (Figure 4C). A similar trend was detected for collagen $\mathrm{X}$, which was positively stained in BMSC and mixed monocultures but only weak in chondrocyte monocultures and co- and tricultures (Figure 4D). Alcian blue staining was used to detect proteoglycan/ aggrecan deposition in the fibrin gel constructs. All culture conditions were uniformly stained blue and showed no differences between monocultures and $\mathrm{OAB}$ co- and tricultures (Figure 4E).

Overall, we observed reduced collagen deposition into the ECM in co- and tricultured fibrin gels during the culture period compared to monocultures.

\section{Biomechanical properties}

We determined the integrity and load capacity of newly generated ECM by measuring its biomechanical properties. Unconfined mechanical testing indicated that $\mathrm{OAB}$ cocultures exhibited a decrease in Young's modulus at 0 to $10 \%$ strain in four of four samples (BMSC) and three of four samples (mixed tricultures and chondrocytes) (Figure 5A). Young's modulus at 40 to $50 \%$ strain showed a decrease in BMSC and mixed OAB co- and tricultures (four of four) while Young's modulus of chondrocytes (three of four) was increased (Figure 5B). Aggregate modulus at equilibrium was reduced in all $\mathrm{OAB}$ coand tricultures compared to monocultures (Figure 5C). Further, three of four BMSC OAB coculture samples had a more than threefold hydraulic permeability compared to monocultures while no clear differences in hydraulic permeability were detected for mixed and chondrocyte $\mathrm{OAB}$ co- and tricultures compared to monocultures (Figure 5D).
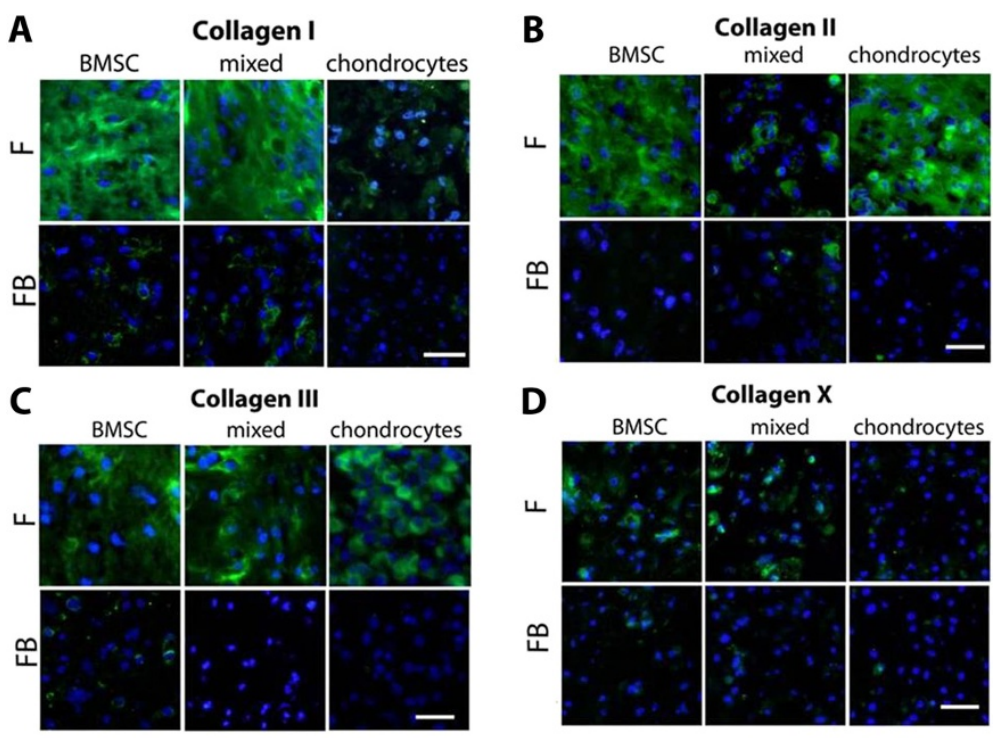

Collagen III

chondrocytes
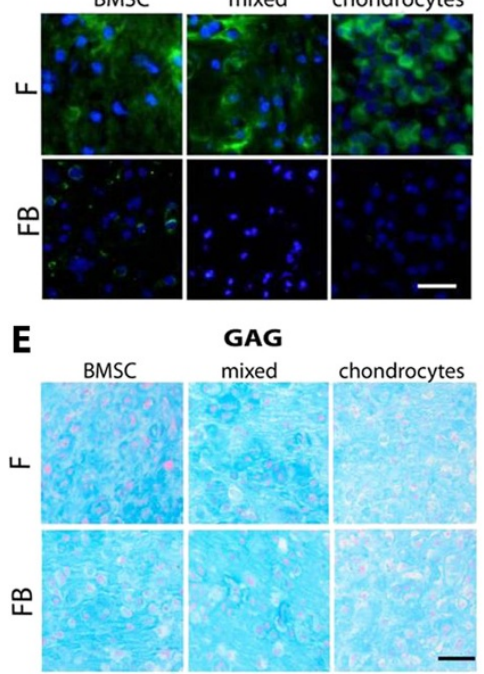

Figure 4 Immunofluorescence staining of collagens. Representative immunofluorescence staining of collagens I (A), II (B), III (C) and X (D) and of Alcian blue (E) in fibrin gels of BMSC, mixed cultures (BMSC + chondrocytes 1:1) and chondrocytes after 28 days of co- and triculture with OAB (FB) or monoculture (F) (Scale bar is $100 \mu \mathrm{m}$ ). BMSC, bone marrow-derived mesenchymal stem cells; F, fibrin gel-embedded monocultures (without subchondral bone explants); FB, fibrin gel-embedded co- and tricultures together with subchondral bone explants; OAB, osteoarthritic subchondral bone. 


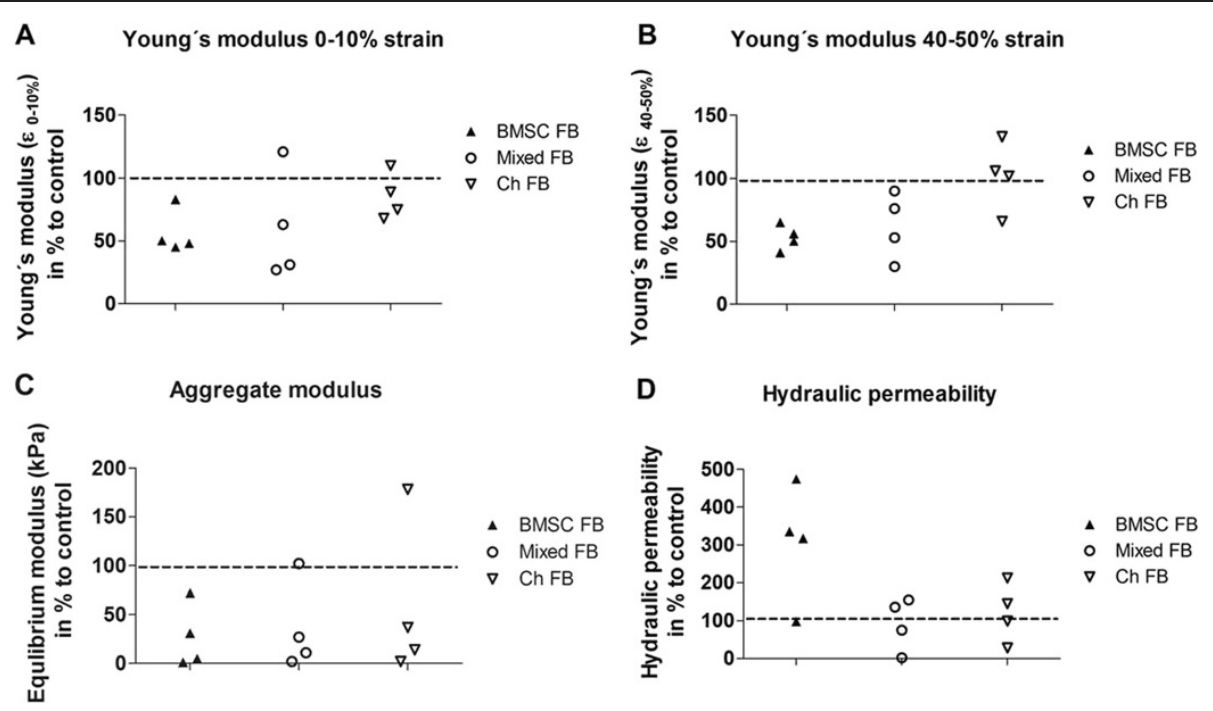

Figure 5 Determination of biomechanical properties. Biomechanical properties of the newly formed ECM at day 28 of BMSC, mixed cultures and chondrocytes co- or tricultured with OAB explants were analyzed and calculated to 100\% of monocultured controls (dotted lines at 100). Young's modulus of (A) 0 to $10 \%$ and (B) 40 to $50 \%$ strain were determined using unconfined compression. Aggregate modulus at equilibrium (C) and hydraulic permeability (D) were determined using confined compression performed at $50 \%$ compression. $N=4$. BMSC, bone marrow-derived mesenchymal stem cells; ECM, extracellular matrix; OAB, osteoarthritic subchondral bone.

\section{Quantification of cytokines, bFGF and GAG in culture supernatants}

In supernatants of ASC, BMSC, mixed cultures and chondrocytes co- or tricultured with $\mathrm{OAB}$ explants, we detected significantly more IL- $1 ß$ at day 28 compared to monocultures whereas NB did not affect IL- $1 ß$ release. In addition, mixed tricultures and chondrocyte cocultures with $\mathrm{OAB}$ released more IL- $1 ß$ into the culture supernatant compared to ASC cocultures with NB (Figure 6A). We detected a significant higher IL-6 level in NB cocultures of ASC compared to all other OAB co- and triculture regimen at day 7 . In addition, all coculture regimens (except for $\mathrm{ASC}+\mathrm{OAB}$ ) released more IL-6 into the culture supernatants compared to monocultures at day 7 (Figure 6B). IL-8 concentrations in supernatant of $\mathrm{NB}$ cocultures with $\mathrm{ASC}$ and $\mathrm{OAB}$ cocultures with BMSC and chondrocytes at day 7 were significantly elevated compared to monocultures. Moreover, IL-8 level in ASC cocultured with $\mathrm{OAB}$ was significant lower than in ASC cocultured with NB (Figure 6C). ASC, BMSC and chondrocyte NB and OAB cocultures released more bFGF into supernatants in comparison to monocultures. In supernatants of $\mathrm{OAB}$ cocultures with BMSC, significantly higher levels of bFGF were detected compared to ASC cocultures with NB (Figure 6D). We detected a significantly higher soluble GAG (sGAG) level in supernatants of all co- and tricultures at day 7 , however, no significant differences between $\mathrm{NB}$ and $\mathrm{OAB}$ cocultures were detectable (Figure 6E). Analysis of supernatants for total soluble collagen content or soluble fibronectin content did not reveal differences (data not shown).
In supernatants of cell-free $\mathrm{OAB}$ and $\mathrm{NB}$ explants, IL- $1 ß$ concentration was in the low $\mathrm{pg} / \mathrm{ml}$ range but was significantly higher in supernatants of $\mathrm{OAB}$ compared to $\mathrm{NB}$ at day 7 . IL-1ß release increased in NB explant supernatants during culture time (Figure 7A). There was no difference with respect to release of IL-6 and IL- 8 between cell-free OAB and NB explants. However, concentration of both cytokines decreased in supernatants during culture time (Figure $7 \mathrm{~B}$ and $\mathrm{C}$ ). bFGF concentration in supernatants of cell-free $\mathrm{OAB}$ explants was significantly higher than in NB explants at both time points. Additionally, bFGF concentration significantly decreased in supernatants of both, OAB and NB explants from day 7 to day 28 (Figure 7D). Cell-free NB explants revealed at both time points a significantly higher level of GAGs in supernatants compared to OAB explants (Figure 7E).

Overall, we observed an increased level of IL-1ß, IL-6, IL-8, bFGF and GAG in supernatants of co- and tricultures. Cell-free NB or OAB explants released these factors indicating an additive effect of bone tissue and co- or tricultured cells. Additionally, we detected differences in cell-free $\mathrm{OAB}$ explants compared to $\mathrm{NB}$ culture supernatants for IL-1ß, bFGF and soluble GAG concentrations.

\section{Stimulation of fibrin gel-embedded monocultures with IL-1ß, IL-6 and IL-8}

To determine whether IL-1ß, IL-6 or IL- 8 has an effect on gene expression, we stimulated BMSC, mixed and chondrocyte monocultures with these cytokines and 


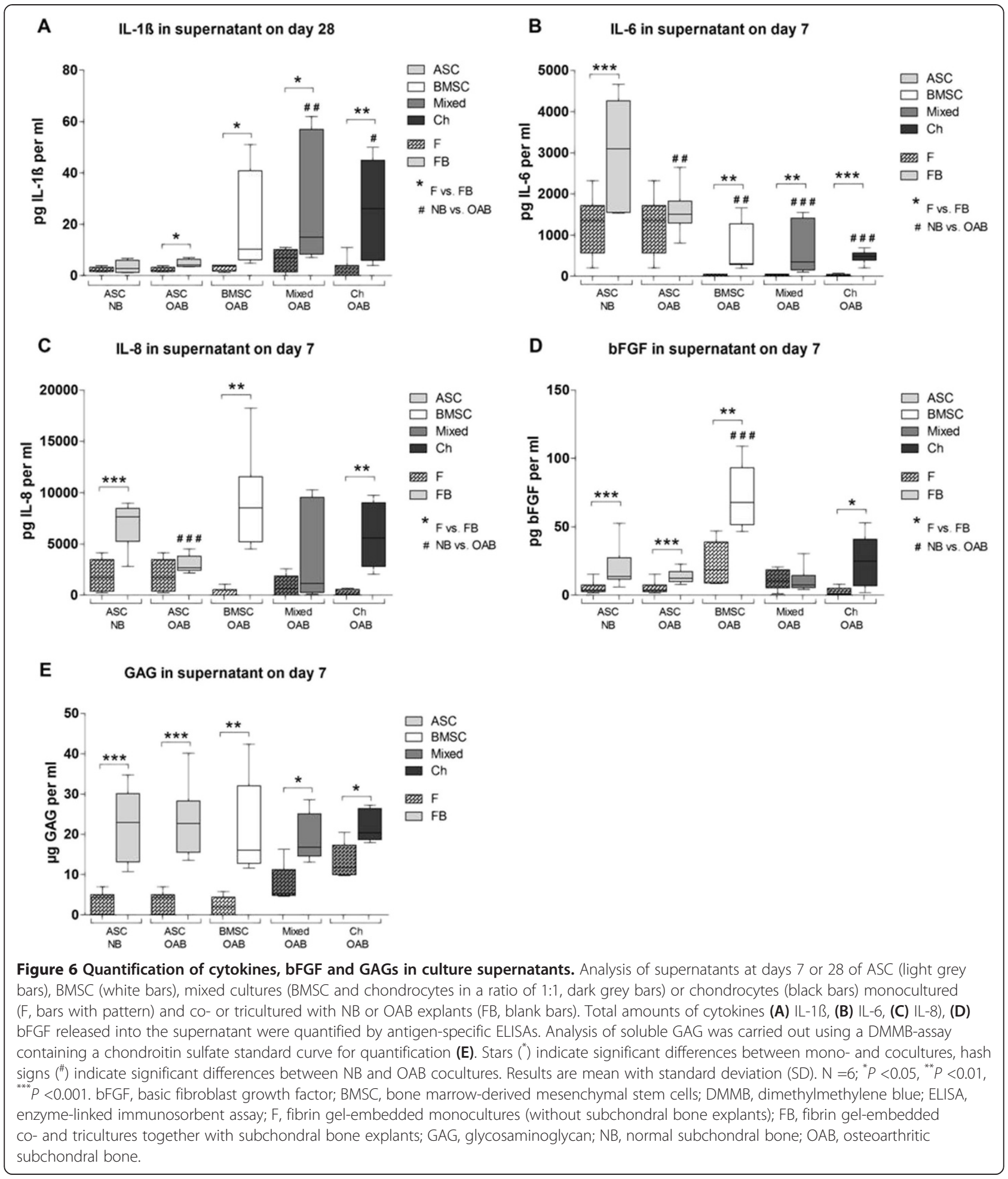

determined mRNA expression of ACAN, MMP2, MMP3 and MMP13 at culture day 7.

We observed a significant inhibition of $A C A N$ gene expression in IL-1ß-stimulated BMSC and mixed cultures in comparison to unstimulated controls. An increase of $M M P 2$ gene expression was detected in IL-1ß-stimulated
BMSC and mixed cultures while $M M P 3$ gene expression was induced in all three culture regimens compared to unstimulated controls. We observed a significant induction of $M M P 13$ gene expression in IL-1ß-stimulated BMSC and chondrocytes whereas MMP13 was downregulated in mixed cultures (Figure 8A). 


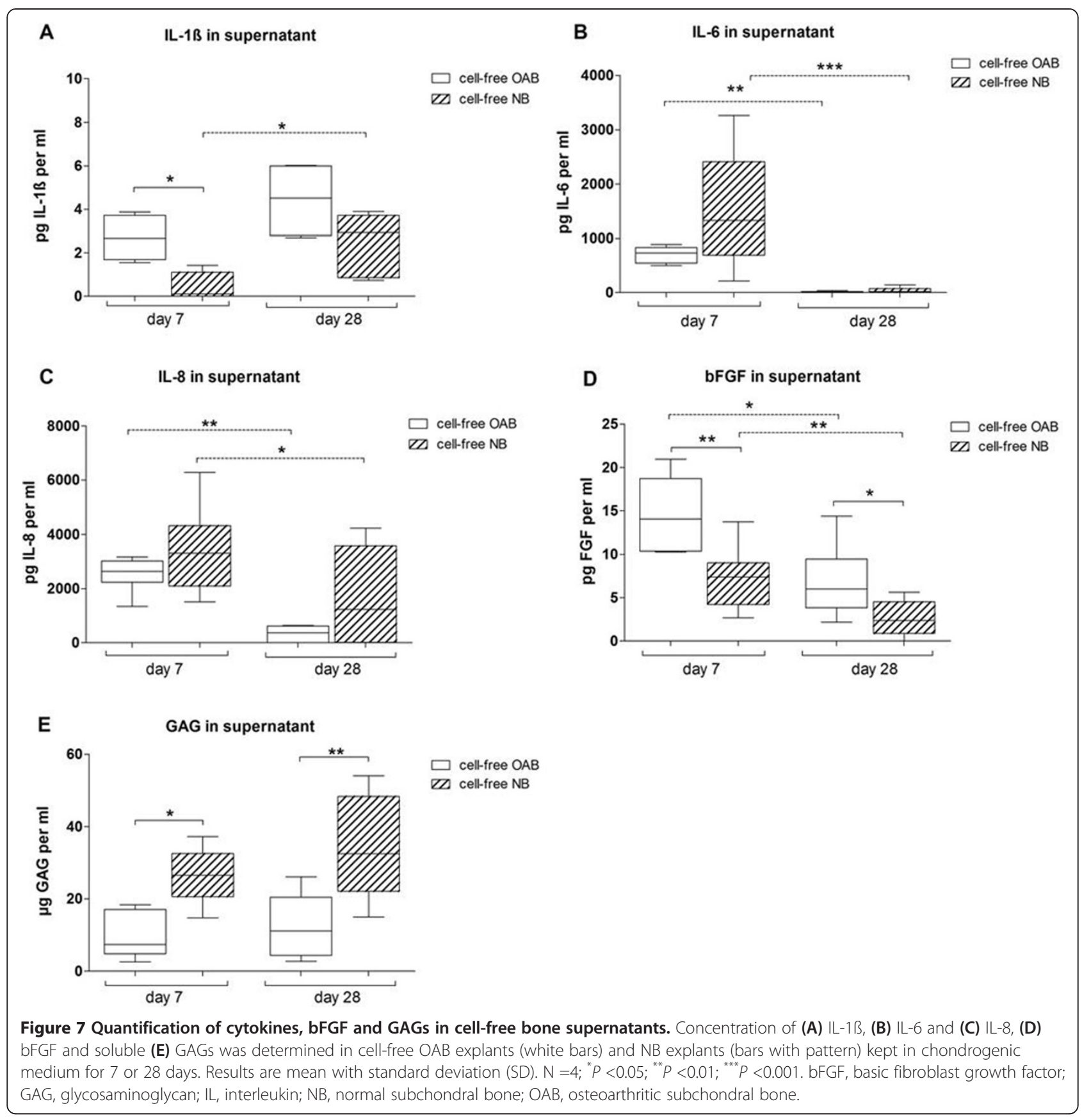

IL-6 stimulation reduced $A C A N$ gene expression in chondrocyte monocultures compared to unstimulated controls only. MMP2 gene expression in all three culture conditions remained unchanged. In contrast, $M M P 3$ expression was downregulated in mixed cultures and chondrocytes in comparison to unstimulated monocultures. IL-6 stimulation reduced MMP13 gene expression in BMSC monocultures compared to unstimulated controls (Figure 8B).

Stimulation with IL-8 induced $A C A N$ gene expression in BMSC. $M M P 2$ gene expression was significantly increased in mixed cultures while MMP3 gene expression in all other culture conditions remained unaffected. Stimulation with IL-8 increased MMP13 gene expression in mixed cultures (Figure $8 \mathrm{C}$ ).

Overall, we observed a mostly inhibitory effect of IL-1ß and IL-6 on gene expression of ACAN. MMP2, MMP3 and $M M P 13$ gene expression was induced mainly by IL-1ß while IL-6 has no effect on $M M P 2$ expression and rather downregulates $M M P 3$ and $M M P 13$ gene expression. IL-8 stimulation has also only little effect on gene expression except for a partly induction of $A C A N, M M P 2$ and $M M P 13$. 


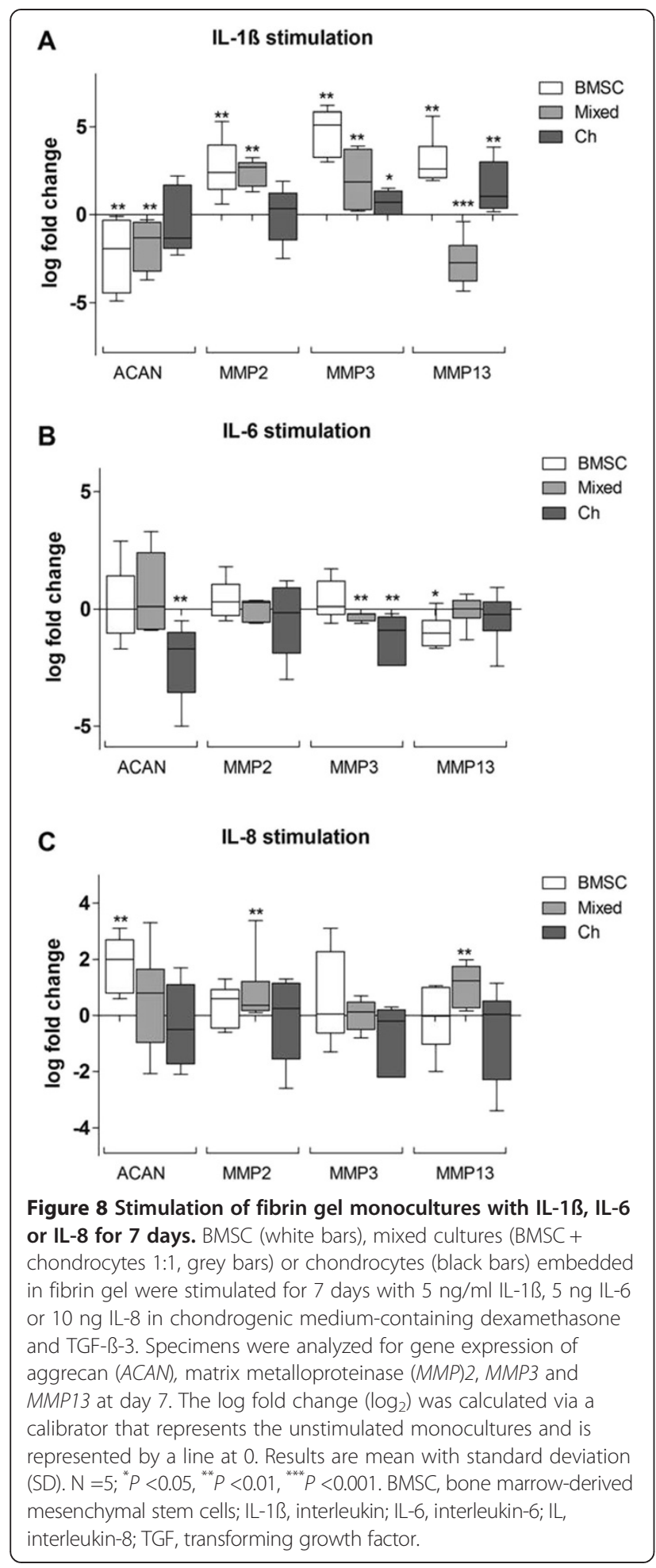

\section{Collagen gene expression of BMSC supplemented with conditioned medium}

Fibrin gel-embedded BMSC monocultures supplemented with $\mathrm{OAB}$ conditioned medium $(\mathrm{CM})$ were analyzed for gene expression of collagens.
At day 7, no differences between BMSC monocultures with and without conditioned medium were observed concerning gene expression of all analyzed collagens. Notably, at day 28 BMSC incubated with CM from OAB revealed a significantly reduced gene expression of all analyzed collagens compared to nonconditioned medium (Figure S3A-D in Additional file 3).

In addition, we detected a significant upregulation of COL2A1 and COL1OA1 gene expression from day 7 to day 28 in nonconditioned cultures whereas COL3A1 was significantly downregulated. Inhibition of COL $2 A 1$ and COL3A1 gene expression at day 28 compared to day 7 was also detected in cultures with CM (Figure S3B-D in Additional file 3).

Overall, we observed a clear inhibitory effect of $O A B$ $\mathrm{CM}$ on gene expression of all collagens at day 28 .

Table S1 in Additional file 4 summarizes all $P$ values indicated in Figures 2, 3, 6, 7, 8 and Figure S3 in Additional file 3.

\section{Discussion}

A so far unmet challenge in the treatment of OA-affected joints is to replace the degraded articular cartilage and subchondral bone matrix with healthy cartilage/bone tissue in order to restore impaired joint function and to prevent or delay total joint replacement by artificial endoprothesis. Cell-based treatment such as autologous chondrocyte implantation (ACI), microfracturing of trauma-induced focal chondral or osteochondral lesions in younger patients prior to establishment of posttraumatic OA is established and leads in general to production of cartilage- and subchondral bone-like replacement tissue $[22,23]$. However, there are conflicting data about longterm stability and functionality of specifically cartilage repair tissue as the composition resembles fibrocartilage rather than hyaline cartilage $[24,25]$. In addition, for older patients or those affected with OA, these treatment options are not successful due to failure or inferior osteochondral defect healing [26]. The reasons for poor healing and repair outcome of cell-based therapies, especially in OA joints, are not comprehensively understood. One likely reason are profoundly altered microenvironmental cues from the surrounding subchondral bone tissue.

In the present study, we analyzed the impact of $O A B$ on chondrogenic differentiation of BMSC, the phenotype of differentiated chondrocytes and matrix-forming capacity. We investigated the influence of $\mathrm{OAB}$ joints on BMSC and chondrocytes assuming that factors produced by OA osteoblasts, osteocytes or osteoclasts can modulate metabolic properties of these cells. It is striking that $O A B$ inhibits gene expression and protein production of collagens I, II, III and X in most co- and triculture setups compared to monocultures. This decrease of collagens is not 
due to increased degradation of existing collagens known to have an in vivo half-life of more than 100 years [27] as a hydroxyproline assay did not reveal increased level of soluble collagens in culture supernatants, indicating that reduced biosynthesis is the major reason for reduced collagen matrix deposition, which in turn causes diminished accumulation of collagen protein in the ECM during the culture time. Since the half-life of collagen mRNA is often only several hours [28], differences in collagen protein level at the culture endpoint are not mandatorily mirrored at mRNA level assessed at the same time.

As synthesis of all investigated collagen types was suppressed in the presence of OAB (albeit collagen $\mathrm{X}$ production was not quantified), no effect specifically on chondrogenic differentiation of BMSC by subchondral bone can be postulated but a general effect on synthesis of matrix macromolecules. Similar inhibitory effects of OA cartilage explants on the production of collagens were described in a recent study by Leyh et al. [29].

When repeating key experiments (gene expression studies and cytokine release) with ASC and NB explants we were unable to detect alterations in gene expression of collagens I and II in coculture with NB suggesting that inhibition, respective alteration of these collagens in ASC cocultures with $\mathrm{OAB}$ is related to factors specific for OA.

The interaction between subchondral bone cells and chondrocytes on the molecular level has not been well understood until now. But there are likely important regulatory events after cartilage injury initiated by subchondral bone cells. In line with our observations, Jiang et al. revealed a suppression of specific markers in cocultures of chondrocytes and osteoblasts like diminished GAG and collagen II deposition or mineralization of ECM [30]. Coculturing of OA chondrocytes with OA osteoblasts induces inhibition of aggrecan production and a concomitant significant increase in $M M P 13$ synthesis. The cocultured osteoblasts also decrease COL2A1, SOX9 and $P T H r P / P T H$-receptor gene expression in the chondrocytes [31,32]. In addition to their data, we observed that in cocultures with subchondral bone (OAB and $\mathrm{NB})$ and in cultures with $\mathrm{OAB} C \mathrm{CM}$ gene expression of COL1OA1 was strongly inhibited compared to monocultures. A specific collagen X-suppressing effect by paracrine coculture of BMSC with healthy articular cartilage was reported earlier from our group and thus appears not to be restricted to bone but to as yet unidentified soluble factor(s) that are common to both tissue types and that are OA independent [19].

Notably, besides reduced synthesis of GAGs in cocultured cell lysates at early time points, we also detected increased sGAGs in supernatants of cocultures. However, as $\mathrm{OAB}$ and $\mathrm{NB}$ cultured without cells also released sGAGs into the supernatant, we believe that we observed an additive effect of cells and explants and not a true increase in degradation of proteoglycans. This is underscored by our observations with ASC cocultures and also by Alcian blue histology, which did not reveal differences between culture conditions at the end of culture (day 28) but at the beginning and by biomechanical properties with respect to permeability and fluid exchange (see below). Therefore, we assume no increase in degradation of proteoglycans and suggest that early inhibition of GAG synthesis is compensated in cocultures during the time line of culture.

We observed an increased release of bFGF in cocultures of BMSC, ASC and chondrocytes with $\mathrm{OAB}$ and in cocultures of ASC with NB explants at the early phase of culture, which is in line with a previous study [33]. As we found also release of bFGF from $\mathrm{OAB}$ and $\mathrm{NB}$ explants cultured without cells, we assume that this adds to the bFGF detected in cocultures. bFGF and other growth factors might be released together with the GAGs they were bound to during pathophysiological situations, that is after tissue injury or during chronic inflammation [34]. Moreover, NB explants released less bFGF compared to $\mathrm{OAB}$ explants indicating a true induction of bFGF release from ASC specifically in the presence of normal bone cells.

Reduced synthesis of matrix components is likely to influence mechanical properties and stability of the resulting matrix. Most important for a successful osteochondral repair is the mechanical stability of the new tissue. The ECM of cartilage consists primarily of three molecules: water, type II collagen and the proteoglycan aggrecan $[35,36]$. Under strain, load is first carried by water, which is displaced from the tissue and leaves the matrix, which carries the load after water has gone from the tissue. Therefore both are physiologically important: water content and its retention in the extrafibrillar matrix together with stability of the fibrillar network. We observed that Young's modulus was reduced in OAB cocultures of BMSC and mixed cultures whereas aggregate modulus was reduced in all cocultures. Hydraulic permeability showed no clear trend for $\mathrm{OAB}$ tricultures and chondrocyte $\mathrm{OAB}$ cocultures. Erickson et al. showed that in every case, BMSC-laden constructs possessed mechanical properties significantly lower than those of chondrocyteseeded hydrogel constructs [37], which is in line with our findings. Our results suggest that coculture with subchondral bone leads to reduced loading capacity of matrix, with increased permeability at least for the BMSC coculture. In contrast, $\mathrm{OAB}$-cocultured chondrocytes showed a trend to increased Young's modulus (depending on strain applied), while hydraulic permeability and aggregate modulus also remained unchanged by subchondral bone coculture. OAB-cocultured chondrocytes seem to produce a matrix with mechanical properties that resemble 
monocultured OA chondrocytes - which does not mean that it is comparable to an ECM synthesized by healthy chondrocytes. Taken together, our data suggest that coculture with $O A B$ impairs mechanical matrix properties with respect to the newly formed fibrillar collagenous network and not of the proteoglycan rich extrafibrillar matrix. This is in line with a previous study from our group, which indicates a distinct modulatory influence of OA cartilage explants that affects the collagen composition of the de novo-produced ECM from co- and tri-cultured cells and leads to impaired mechanical and biochemical properties of the matrix because of an altered fibrillar network [29].

Although many studies have examined effects of factors released from cartilage and chondrocytes on chondrogenesis, only few have focused on the influence of proinflammatory cytokines from subchondral bone on the chondrogenic differentiation and matrix-forming capacity of BMSC [38,32]. In the current study, we analyzed proinflammatory cytokines like IL-1ß, IL-6 and IL-8, which are known to be released from BMSC and OA chondrocytes and are considered to contribute to OA pathogenesis $[39,40]$. We discovered that coculture with $\mathrm{OAB}$ truly induced the concentration of these cytokines/ chemokines in culture supernatants in all culture conditions. This was most prevalent in the early phase of the culture except for IL-1ß, which remained induced throughout the culture time. Notably, comparison of inflammatory factors released by $\mathrm{OAB}$ and $\mathrm{NB}$ explants in cell-free and coculture experiments revealed differences. First, cell-free NB explants released less IL- $1 ß$ and bFGF but more GAGs than OAB and - importantly - NB did not induce IL- $1 ß$ release in coculture. Second, IL- 6 and (partly) IL-8 release in cocultures with NB explants was higher than release from $\mathrm{OAB}$ cocultures. Presumably, these metabolic differences result from a differently composed ECM of bone tissue from younger trauma patients, including increased osteoblast numbers and activity resulting in a higher bone-forming capacity compared to older OAB [41,42]. We suggest that these cytokines in part mediate suppression of collagen and GAG synthesis and induce GAG release from proteoglycans, which would be in line with data from literature $[32,43]$. To prove our hypothesis, we stimulated monocultures of BMSC, mixed cells and chondrocytes - embedded in fibrin gel - with IL-1ß, IL-6 or IL-8. We observed that stimulation with IL-1ß in general induced $M M P 2, M M P 3$ and $M M P 13$ gene expression, which all are important players with respect to matrix degradation. Additionally, IL-1ßstimulation of BMSC and of mixed cultures leads to a significant downregulation of $A C A N$ gene expression, which is in line with most recent literature [39], however, Salter et al. reported involvement of IL-1ß in a chondroprotective signaling cascade, which is defined by increasing ACAN mRNA in chondrocytes [44]. In concert with
IL-1ß, IL-6 inhibits proteoglycan production and can in addition amplify IL-1ß effects [39]. We observed that IL-6 effects on $A C A N$ gene expression were less pronounced as after IL-1ß stimulation. MMP2 expression was not affected while $M M P 3$ and $M M P 13$ expression were mostly downregulated after IL-6 stimulation. This is in line with studies that predict that IL-6 might have also a beneficial role in OA pathology. OA chondrocytes produce more IL-6 during cartilage regeneration than healthy chondrocytes. A recent study of Tsuchida et al. revealed a modest anabolic role for IL-6 in cartilage matrix regeneration demonstrating that chondrocytes produce high amounts of IL-6, which is released into the synovial fluid and has possible beneficial effects on cartilage regeneration [45]. It appears that chondrocytes react more sensitive to OA conditions as we observed no differences in IL-6 and IL-8 level in OAB versus NB bone explant supernatants.

We demonstrated inductive effects of IL-8 on gene expression of mixed cultures for $M M P 2$ and $M M P 13$ and of BMSC for ACAN, while chondrocytes did not respond. IL-8 initiates chemotaxis in cells and leads to cell migration aligned with $M M P$ upregulation. This could be interpreted as a trial for cartilage repair with local BMSC and suggests, that IL-8 is required to initiate tissue repair in vivo [46]. Notably, chondrocytes seemed to be not affected by IL- 8 stimulation possibly, because OA chondrocytes are less sensitive to cytokine stimulation than normal cells [47].

In addition, we wanted to answer the question if differentiated chondrocytes, which are in close cell-to-cell contact with undifferentiated BMSC ameliorate or augment inhibitory effects of subchondral bone explants on BMSC. Overall, we observed that collagen gene expression was mostly unaltered while protein expression and biomechanical properties of $\mathrm{OAB}$ tricultured mixed populations resemble rather $\mathrm{OAB}$ cocultured BMSC than chondrocytes. We thus suggest that chondrocytes in the mixed cultures do not alter BMSC metabolism in tricultures with subchondral bone explants and that response of mixed cultures to factors from OAB resemble rather that of cocultured BMSC than that of cocultured chondrocytes.

In order to include a 'reference' for BMSC that were all obtained from OA-affected donors, we repeated key experiments with ASC which served as 'healthy' controls. We did not find striking differences between both cell types with respect to influence of coculture on collagen gene expression and cytokine, bFGF and GAG release. Thus we suggest that tissue source (ASC versus $\mathrm{BMSC}$ ) and age and disease status (OA versus non-OA) of cell donors do not affect matrix-forming capacity and chondrogenic differentiation at least under in vitro conditions. 
In order to decide if observed effects are related to OA, we have repeated key experiments with subchondral bone from trauma patients. We observed differences in release of IL-1ß, bFGF and GAG between OAB and NB cell-free explants and also with respect to gene expression and cytokine release in $\mathrm{OAB}$ versus $\mathrm{NB}$ cocultures. However, a limitation of our study is caused by the control group, which consists of trauma patients who have a lower mean age than those individuals with OA included into the study group. Therefore, differences in cytokine release as we have observed between $\mathrm{NB}$ and $\mathrm{OAB}$ might result not only from $\mathrm{OA}$ pathogenesis but also from different metabolic activities due to the age of the bone donor. In the current study, it is thus not possible to assign these metabolic alterations specifically to OA as age of bone donors might have also an impact.

\section{Conclusions}

We observed elevated soluble factors in coculture supernatants compared to monocultures in parallel to a metabolic shift in cocultured BMSC and OA chondrocytes, which affects synthesis of collagens and proteoglycans. This reduced biosynthesis of structural macromolecules alters composition of the fibrillar collagen networks of the ECM and impairs mechanical stability and integrity of the newly formed matrix. However, impaired production of proteoglycans appears to be compensated during culture time and thus not to be responsible for inferior mechanical properties of the ECM.

We suggest that these effects are at least partly mediated by IL-1ß, bFGF and IL-6, which are increased in coculture supernatants while IL-8 effects are less pronounced with respect to investigated parameters.

Our data imply that implanting BMSC or chondrocytes into subchondral bone lesions or onto the surface of denuded OA bone will not result in formation or regeneration of a well functional cartilage-like matrix. Thus before trying to restore eroded cartilage tissue in an OA-affected joint by a cell-based approach, release of proinflammatory cytokines and additional soluble factors as GAGs and growth factors from surrounding tissues has to be strictly controlled.

\section{Additional files}

Additional file 1: Figure S2. FACS analysis of BMSC surface markers and staining of adipogenic and osteogenic differentiated BMSC. (A) Isolated plastic adherent BMSC in passage 3 were analyzed by flow cytometry using specific antibodies against BMSC negative markers CD 19 and CD 34 and BMSC positive markers CD 44 and CD 105 (red line). Specificity of epitope staining was proofed using an isotype control (black line). (B) BMSC were stained with oil red for oil drops incorporated during adipogenic differentiation (upper row) and with alizarin red for matrix mineralization during osteogenic differentiation (lower row).
Additional file 2: Figure S1. Vitality of fibrin gel-embedded cells and coculture setups. Vitality of (A) BMSC, (B) mixed cultures (BMSC and chondrocytes in a ratio of $1: 1)$ and $(\mathbf{C})$ chondrocytes was determined in monocultures (F) and co- or tricultures with $\mathrm{OAB}(\mathrm{FB})$ kept in chondrogenic medium. Content of LDH was quantified in the supernatant of days 7, 14, 21 and 28 and compared to assay controls (high control = all cells in fibrin gels were lysed; low control = spontaneous cell death of an equivalent cell amount in monolayer). Due to high interexperimental variability, we have calculated the raw data as a percentage of control per individual experiment. Results are mean with standard variation (SD). $N=4 ;{ }^{*} P<0.05$, ${ }^{* *} P<0.01,{ }^{* * *} P<0.001$.

Additional file 3: Figure S3. Quantification of gene expression in BMSC monocultures stimulated with $\mathrm{OA}$ bone explants conditioned medium. Gene expression level of (A) COL1A1, (B) COL2A1, (C) COL3A1 and (D) COL10A1 were determined in monocultures supplemented with OABconditioned medium using plasmid standard curves. BMSC monoculture with fresh medium ( $F$, bars with pattern) and monoculture of BMSC supplemented with $\mathrm{OAB}$ conditioned chondrogenic medium ( $F+C M$, blank bars) were analyzed after 7 or 28 days. Due to high interexperimental variability we have calculated the raw data as a percentage of highest CDNA copy number per individual experiment. Solid lines indicate significant differences between culture conditions, dotted lines indicate significant differences between culture time points (days 7 and 28). $N=4 ;^{*} P<0.05$, ${ }^{* *} P<0.01,{ }^{* * *} P<0.001$.

Additional file 4: Table S1. Statistical data ( $P$ values). $P$ values of: (A) Cocultures vs. monocultures and normal vs. OA subchondral bone cocultures. COL1A1, COL2A1, COL3A1 and COL1OA1 gene expression determined in ASC, BMSC, mixed cultures or chondrocytes kept as monocultures (F) or as cO- and tricultures with osteoarthritic (OA FB) or normal subchondral bone explants (NB FB) at day 7 and day 28 (corresponding to Figure 2). (B) Cocultures vs. monocultures. Collagen protein (day 28) and proteoglycan (day 7) quantification in cell lysates from BMSC, mixed cultures or chondrocytes kept as monocultures (F) or as co- and tricultures with OA subchondral bone explants (FB) (corresponding to Figure 3).

\section{Abbreviations}

ACAN: aggrecan gene; ACl: autologous chondrocyte implantation; ASC: adipose-derived stem cells; bFGF: basic fibroblast growth factor; BMSC: bone marrow-derived mesenchymal stem cells; BSA: bovine serum albumin; CM: conditioned medium; Col10A1: gene collagen $X_{;}$COL1A1: gene collagen I; COL2A1: gene collagen II; COL3A1: gene collagen III;

DMEM: Dulbecco's modified Eagle's medium; DMMB: dimethylmethylene blue; ECM: extracellular matrix; ELISA: enzyme-linked immunosorbent assay; FCS: fetal calf serum; GAG: glycosaminoglycan; IL-1ß: interleukin 1ß; IL6: interleukin 6; IL-8: interleukin 8; LDH: lactate dehydrogenase; MMP: matrix metalloproteinase; NB: normal subchondral bone; OA: osteoarthritis; OAB: OA subchondral bone; PBS: phosphate-buffered saline; PFA: paraformaldehyde; TBS: Tris-buffered saline; TEP: total knee replacements; PTHrP/PTH: parathyroid hormone-related protein/parathyroid hormone receptor; TGF-ß: transforming growth factor $B$.

\section{Competing interests}

The authors declare that they have no competing interests.

\section{Authors' contributions}

ML performed gene expression analysis, histology and immunofluorescence stainings and all biochemical assays and contributed with data evaluation. AS performed all biomechanical tests and helped to draft the manuscript. LD evaluated biomechanical tests and helped to draft and to revise the manuscript. JS acquired BMSC, cartilage and bone explants and performed cell culture and cell isolation from bone and cartilage tissue. He helped to revise the manuscript. Al has made contributions to conception and design of the study and drafting and revising the manuscript. JG has made contributions to conception and design of the study and drafting and revising the manuscript. SG, as corresponding author, is responsible for conception, coordination and design of the study and has written, drafted and critically revised the manuscript. All authors read and approved the final manuscript. 


\section{Acknowledgements}

This study was supported by funds from the German Research Foundation (DFG) (GR 1301/8-1) and from the German Society for Orthopedics and Orthopedic Surgery (DGOOC). The authors are indebted to Lilly Weger for her excellent technical assistance. Adipose-derived stem cells were a friendly gift from the Department of Otorhinolaryngology, Division of Facial Plastic Surgery, University of Regensburg. Additionally, we thank Robert H. Springorum for being an abundant source for surgical collected material. The CIIC1 antibody developed by R. Holmdahl and K. Rubin was obtained from the Developmental Studies Hybridoma Bank developed under the auspices of the NICHD and maintained by The University of lowa.

\section{Author details}

${ }^{1}$ Department of Orthopedic Surgery, University of Regensburg, ZMB/BioPark 1, Josef-Engert-Str. 9, Regensburg 93053, Germany. ${ }^{2}$ Centre for Medical Biotechnology, BioPark 1, Josef-Engert-Str. 9, Regensburg 93053, Germany. ${ }^{3}$ Institute of Orthopedic Research and Biomechanics, Centre of Musculoskeletal Research, University of Ulm, Helmholtzstr. 14, Ulm 89081, Germany.

Received: 27 November 2013 Accepted: 5 September 2014 Published online: 07 October 2014

\section{References}

1. van den Berg WB: Osteoarthritis year 2010 in review: pathomechanisms. Osteoarthritis Cartilage 2011, 19:338-341.

2. Goldring MB, Marcu KB: Cartilage homeostasis in health and rheumatic diseases. Arthritis Res Ther 2009, 11:224.

3. Karsdal MA, Leeming DJ, Dam EB, Henriksen K, Alexandersen P, Pastoureau P, Altman RD, Christiansen C: Should subchondral bone turnover be targeted when treating osteoarthritis? Osteoarthritis Cartilage 2008, 16:638-646.

4. Felson DT, Neogi T: Osteoarthritis: is it a disease of cartilage or of bone? Arthritis Rheum 2004, 50:341-344.

5. Mansell JP, Tarlton JF, Bailey AJ: Biochemical evidence for altered subchondral bone collagen metabolism in osteoarthritis of the hip. BrJ Rheumatol 1997, 36:16-19.

6. Funck-Brentano T, Cohen-Solal M: Crosstalk between cartilage and bone: when bone cytokines matter. Cytokine Growth Factor Rev 2011, 22:91-97.

7. Pittenger MF, Mackay AM, Beck SC, Jaiswal RK, Douglas R, Mosca JD, Moorman MA, Simonetti DW, Craig S, Marshak DR: Multilineage potential of adult human mesenchymal stem cells. Science 1999, 284:143-147.

8. Kolf CM, Cho E, Tuan RS: Mesenchymal stromal cells. Biology of adult mesenchymal stem cells: regulation of niche, self-renewal and differentiation. Arthritis Res Ther 2007, 9:204.

9. Djouad F, Delorme B, Maurice M, Bony C, Apparailly F, Louis-Plence P, Canovas F, Charbord P, Noel D, Jorgensen C: Microenvironmental changes during differentiation of mesenchymal stem cells towards chondrocytes. Arthritis Res Ther 2007, 9:R33.

10. Birmingham E, Niebur GL, McHugh PE, Shaw G, Barry FP, McNamara LM: Osteogenic differentiation of mesenchymal stem cells is regulated by osteocyte and osteoblast cells in a simplified bone niche. Eur Cell Mater 2012, 23:13-27.

11. Bian L, Zhai DY, Mauck RL, Burdick JA: Coculture of human mesenchymal stem cells and articular chondrocytes reduces hypertrophy and enhances functional properties of engineered cartilage. Tissue Eng Part $A$ 2011, 17:1137-1145

12. Grassel S, Ahmed N: Influence of cellular microenvironment and paracrine signals on chondrogenic differentiation. Front Biosci 2007, 12:4946-4956.

13. Westacott Cl, Webb GR, Warnock MG, Sims JV, Elson CJ: Alteration of cartilage metabolism by cells from osteoarthritic bone. Arthritis Rheum 1997, 40:1282-1291.

14. Mo XT, Guo SC, Xie HQ, Deng L, Zhi W, Xiang Z, Li XQ, Yang ZM: Variations in the ratios of co-cultured mesenchymal stem cells and chondrocytes regulate the expression of cartilaginous and osseous phenotype in alginate constructs. Bone 2009, 45:42-51.

15. Thompson AD, Betz MW, Yoon DM, Fisher JP: Osteogenic differentiation of bone marrow stromal cells induced by coculture with chondrocytes encapsulated in three-dimensional matrices. Tissue Eng Part A 2009, 15:1181-1190
16. Ahmed TA, Dare EV, Hincke M: Fibrin: a versatile scaffold for tissue engineering applications. Tissue Eng Part B Rev 2008, 14:199-215.

17. Geyer M, Grassel S, Straub RH, Schett G, Dinser R, Grifka J, Gay S, Neumann E, Muller-Ladner U: Differential transcriptome analysis of intraarticular lesional vs intact cartilage reveals new candidate genes in osteoarthritis pathophysiology. Osteoarthritis Cartilage 2009, 17:328-335.

18. Gehmert S, Hidayat M, Sultan M, Berner A, Klein S, Zellner J, Muller M, Prantl L: Angiogenesis: the role of PDGF-BB on adipose-tissue derived stem cells (ASCs). Clin Hemorheol Microcirc 2011, 48:5-13.

19. Ahmed N, Dreier R, Gopferich A, Grifka J, Grassel S: Soluble signalling factors derived from differentiated cartilage tissue affect chondrogenic differentiation of rat adult marrow stromal cells. Cell Physiol Biochem 2007, 20:665-678

20. Mow VC, Kuei SC, Lai WM, Armstrong CG: Biphasic creep and stress relaxation of articular cartilage in compression? Theory and experiments. J Biomech Eng 1980, 102:73-84.

21. Frank RS, Hochmuth RM: An investigation of particle flow through capillary models with the resistive pulse technique. J Biomech Eng 1987, 109:103-109.

22. Brittberg $M$, Lindahl A, Nilsson A, Ohlsson C, Isaksson O, Peterson L: Treatment of deep cartilage defects in the knee with autologous chondrocyte transplantation. N Engl J Med 1994, 331:889-895.

23. Marlovits S, Zeller $P$, Singer $P$, Resinger $C$, Vecsei V: Cartilage repair: generations of autologous chondrocyte transplantation. Eur J Radiol 2006, 57:24-31.

24. Peterson L, Vasiliadis HS, Brittberg M, Lindahl A: Autologous chondrocyte implantation: a long-term follow-up. Am J Sports Med 2010, 38:1117-1124.

25. Vanlauwe J, Saris DB, Victor J, Almqvist KF, Bellemans J, Luyten FP: Five-year outcome of characterized chondrocyte implantation versus microfracture for symptomatic cartilage defects of the knee: early treatment matters. Am J Sports Med 2011, 39:2566-2574.

26. de Windt TS, Bekkers JE, Creemers LB, Dhert WJ, Saris DB: Patient profiling in cartilage regeneration: prognostic factors determining success of treatment for cartilage defects. Am J Sports Med 2009, 37:58S-62S.

27. Verzijl N, DeGroot J, Thorpe SR, Bank RA, Shaw JN, Lyons TJ, Bijlsma JW, Lafeber FP, Baynes JW, TeKoppele JM: Effect of collagen turnover on the accumulation of advanced glycation end products. J Biol Chem 2000, 275:39027-39031.

28. Dozin B, Quarto R, Rossi F, Cancedda R: Stabilization of the mRNA follows transcriptional activation of type II collagen gene in differentiating chicken chondrocyte. J Biol Chem 1990, 265:7216-7220.

29. Leyh M, Seitz A, Durselen L, Springorum HR, Angele P, Ignatius A, Grifka J, Grassel S: Osteoarthritic cartilage explants affect extracellular matrix production and composition in cocultured bone marrow-derived mesenchymal stem cells and articular chondrocytes. Stem Cell Res Ther 2014, 5:77.

30. Jiang J, Nicoll SB, Lu HH: Co-culture of osteoblasts and chondrocytes modulates cellular differentiation in vitro. Biochem Biophys Res Commun 2005, 338:762-770.

31. Sanchez C, Deberg MA, Piccardi N, Msika P, Reginster JY, Henrotin YE: Subchondral bone osteoblasts induce phenotypic changes in human osteoarthritic chondrocytes. Osteoarthritis Cartilage 2005, 13:988-997.

32. Sanchez C, Deberg MA, Piccardi N, Msika P, Reginster JY, Henrotin YE: Osteoblasts from the sclerotic subchondral bone downregulate aggrecan but upregulate metalloproteinases expression by chondrocytes. This effect is mimicked by interleukin- 6 , -1 beta and oncostatin M pre-treated non-sclerotic osteoblasts. Osteoarthritis Cartilage 2005, 13:979-987.

33. Fischer J, Dickhut A, Rickert M, Richter W: Human articular chondrocytes secrete parathyroid hormone-related protein and inhibit hypertrophy of mesenchymal stem cells in coculture during chondrogenesis. Arthritis Rheum 2010, 62:2696-2706.

34. D'Amore PA: Modes of FGF release in vivo and in vitro. Cancer Metastasis Rev 1990, 9:227-238.

35. Hollander AP, Dickinson SC, Kafienah W: Stem cells and cartilage development: complexities of a simple tissue. Stem Cells 2010, 28:1992-1996.

36. Poole AR, Kojima T, Yasuda T, Mwale F, Kobayashi M, Laverty S: Composition and structure of articular cartilage: a template for tissue repair. Clin Orthop Relat Res 2001, 391S:S26-S33. 
37. Erickson IE, Huang AH, Chung C, Li RT, Burdick JA, Mauck RL: Differential maturation and structure-function relationships in mesenchymal stem cell- and chondrocyte-seeded hydrogels. Tissue Eng Part A 2009, 15:1041-1052.

38. Goldring MB, Goldring SR: Osteoarthritis. J Cell Physiol 2007, 213:626-634.

39. Fernandes JC, Martel-Pelletier J, Pelletier JP: The role of cytokines in osteoarthritis pathophysiology. Biorheology 2002, 39:237-246.

40. Westacott Cl, Sharif M: Cytokines in osteoarthritis: mediators or markers of joint destruction? Semin Arthritis Rheum 1996, 25:254-272.

41. Luder HU: Age changes in the articular tissue of human mandibular condyles from adolescence to old age: a semi quantitative light microscopic study. Anat Rec 1998, 251:439-447.

42. Kouri JB, Lavalle C: Do chondrocytes undergo "activation" and "transdifferentiation" during the pathogenesis of osteoarthritis? A review of the ultrastructural and immunohistochemical evidence. Histol Histopathol 2006, 21:793-802.

43. Ryu JH, Chun JS: Opposing roles of WNT-5A and WNT-11 in interleukin-1 beta regulation of type II collagen expression in articular chondrocytes. J Biol Chem 2006, 281:22039-22047.

44. Salter DM, Millward-Sadler SJ, Nuki G, Wright MO: Differential responses of chondrocytes from normal and osteoarthritic human articular cartilage to mechanical stimulation. Biorheology 2002, 39:97-108.

45. Tsuchida Al, Beekhuizen M, Rutgers M, van Osch GJ, Bekkers JE, Bot AG, Geurts B, Dhert WJ, Saris DB, Creemers LB: Interleukin-6 is elevated in synovial fluid of patients with focal cartilage defects and stimulates cartilage matrix production in an in vitro regeneration model. Arthritis Res Ther 2012, 14:R262.

46. Mishima Y, Lotz M: Chemotaxis of human articular chondrocytes and mesenchymal stem cells. J Orthop Res 2008, 26:1407-1412.

47. Fan Z, Bau B, Yang H, Soeder S, Aigner T: Freshly isolated osteoarthritic chondrocytes are catabolically more active than normal chondrocytes, but less responsive to catabolic stimulation with interleukin-1beta. Arthritis Rheum 2005, 52:136-143.

doi:10.1186/s13075-014-0453-9

Cite this article as: Leyh et al: Subchondral bone influences

chondrogenic differentiation and collagen production of human bone marrow-derived mesenchymal stem cells and articular chondrocytes. Arthritis Research \& Therapy 2014 16:453.

\section{Submit your next manuscript to BioMed Central and take full advantage of:}

- Convenient online submission

- Thorough peer review

- No space constraints or color figure charges

- Immediate publication on acceptance

- Inclusion in PubMed, CAS, Scopus and Google Scholar

- Research which is freely available for redistribution 